\title{
The development and standardization of an Indian Positive Body Image Scale with an Exploratory Research Design
}

\author{
Pankaj Jain ${ }^{1}$ and Gyanesh Kumar Tiwari ${ }^{1}$ \\ ${ }^{1}$ Department of Psychology, School of Humanities \& Social Sciences, Doctor Harisingh \\ Gour Vishwavidyalaya, Sagar, 470003, Madhya Pradesh, India
}

May 18, 2020

\begin{abstract}
Background: The study explored the basic features of positive body image intending to standardize a scale for it employing an Exploratory Research Design.

Participants and Procedure: The study was conducted in two phases. In the first phase, 17 males and 18 females with age range 21 to 30 years were chosen as per the screening criteria based on the scores of body mass index and body appreciation scale. The semi-structured interviews were conducted followed by its verbatim inscription for developing themes and basic descriptions of positive body image. The second phase involved 507 participants (268 males and 239 females) with the age span of 20 to 35 years on whom the newly developed positive body image scale and general health and quality of life measures meant for validation were administered

Results: A phenomenon with complex facets, linked with self, social realities and life outcomes, health practices and social relevance were the major themes generated in the first phase. The second phase resulted in the development of a new positive body image scale with 24 items comprising two components namely body appreciation and body effectiveness with desirable psychometric properties.

Conclusions: A set of theoretically sound and practically relevant attributes of positive body image was identified. The test-retest reliability showed significant levels while coefficients of correlations of the derived measure with general health and quality of life represented its validity. Further verifications of the findings are recommended. The study may carry implications for researchers, practitioners and policymakers.
\end{abstract}

\section{Keywords}

positive body image, body appreciation, body effectiveness, general health, exploratory research design.

\section{Highlights}

$\varnothing$ We explored the basic themes and attributes through a qualitative study.

$\varnothing$ The present study employed an exploratory research design, a variant of mixed methods design.

$\varnothing$ We standardized a 24 items positive body image scale based on the Indian adults.

$\varnothing$ The scale evinced the commendable psychometric properties and predictive strengths.

\section{Introduction}

Self-concept constitutes an important component of human personality which plays a pivotal role in the development, regulation and maintenance of the nature and dynamics of human behaviours. Body 
image is an inseparable constituent of self-concept which significantly influences the human functioning, performance and quality of life (Cash \& Smolak, 2011). Body image has been reported to regulate and impact interpersonal relationships, self-esteem, eating behaviours, well-being and other dimensions of human behaviours irrespective of age, gender, cultural background and personality attributes of the individuals. Recent years have witnessed an upsurge in the diversity and sophistication in body image scholarship comprising body image development and difficulties of boys and girls from various cultures along with new assessment tools.

The body image scholarship has taken a new shape after the emergence of positive psychology as a new branch of Psychology in 2000. Before that, the scientific study of body image was basically confined to the efforts to understand negative body image especially with reference to the eating disorders and other emotional problems of women. The researchers have demonstrated that body image has significant implications for understanding human behaviours and performance in a multitude of individual, interpersonal and social contexts. It has been argued that the construct of positive body image is multi-faceted and multi-dimensional in nature, different from the negative body image, characterized by different attributes and regulatory mechanisms, closely associated with a host of measures namely body appreciation, positive rational acceptance coping, body image flexibility, body functionality, attunement, body pride, positive and self-accepting body talk, body sanctification, a broad conceptualization of beauty and body acceptance by others (Webb et al., 2015). The positive body image has also been reported to play a significant role in positive human functioning and productivity proliferating across age, gender and cultures (Jain \& Tiwari, 2016b; Webb et al., 2015).

The has been argued that the scientific study of positive body image attracted less attention in comparison to negative body image (Avalos et al., 2005; Tylka \& Wood-Barcalow, 2015) involving different types of psychopathologies such as eating disorders, dysmorphic disorder etc., employing mostly women participants having affiliations with the Western socio-cultural milieu. It has also been argued that the majority of the researches have been carried out applying quantitative methods which have failed in their attempts to conceptualize the exact nature and dynamics of positive and negative body image. The researchers have suggested that future studies should focus to employ qualitative methods or mixed methods which would decipher the true nature of body image of any kind (Tylka \& Wood-Barcalow, 2015).

The scales of positive body image like the Body Esteem Scale (Franzoi \& Shields, 1984), the Body Esteem Scale for Adolescents and Adults (Mendelson et al., 2001), the Appearance Evaluation subscale of the Multidimensional Body-Self Relations Questionnaire (Brown et al., 1990; Cash, 2002) and body appreciation scale (Avalos et al., 2005) have been based on satisfaction. In addition, these scales have conceptualized body image as a continuous bipolar construct with positive and negative poles representing body satisfaction and body dissatisfaction, respectively (Webb et al., 2015). The recent findings have shown that positive and negative body images are different and regulated by dissimilar dynamics (Tylka \& Wood-Barcalow, 2015).

The researchers have suggested for developing a new measure for cultivating a deeper understanding into the nature and dynamics of positive body image employing qualitative and mixed methods study of diverse samples with dissimilar socio-cultural features (Webb et al., 2015). It has also been observed that initial qualitative studies of positive body image have exerted formative influences on the conceptualizations and measurement of positive body image (Frisén \& Holmqvist, 2010; Holmqvist \& Frisén, 2012; Wood-Barcalow et al., 2010a). In addition, these studies have helped to understand and establish curative, preventive and promotive potentials of positive body image (Webb et al., 2015).

\section{Present Investigation}

The present research attempted to explore the basic nature and attributes of positive body image with the ultimate goal to standardize a scale of positive body image based on the inherent contents of the qualitative data. The study employed an exploratory mixed methods design which comprised data collection in two phases; the qualitative phase and the quantitative phase with different but mutually inclusive 
objectives. Based on the insights from emerged themes and sub-themes of the qualitative phase of the study, a comprehensive positive body image scale was standardized in the second phase of the study.

Researchers have analyzed interviews of the participants having positive body image which resulted in the emergence of features such as body appreciations, body acceptance and love, inner positivity, influencing outer demeanour, a broad conceptualization of beauty, media literate, unconditional acceptance from others, searching for others with a positive body image, spirituality, and body care irrespective of many differences (Frisén \& Holmqvist, 2010; Wood-Barcalow et al., 2010b). These constitute core features of positive body image that promote and maintain it. It has also a reciprocal impact on the environment in a growth-enhancing manner.

Measurement of positive body image has been recognized as an important task before researchers. Several positive body image scales have been developed. The empirical evidence suggested that none of these tools focused on the attributes of positive body image in depth. Therefore, it necessitated developing a comprehensive measure of positive body image based on the Indian population. It has been suggested by previous researchers that the exploratory design, a variant of mixed methods design, is very helpful where the nature and attributes of the construct/s being studied are not explicit or established, the measures or instruments have not been standardized, the significant correlates are not known or there is no guiding framework or theory (Creswell et al., 2003; Creswell, 2004). In this backdrop, it was aimed to develop a scale to measure positive body image on Indian Population in the present study. The researchers have reported that research on body image applying qualitative studies is lacking and qualitative or mixed methods have been suggested to explicate the true nature of positive body image (Webb et al., 2015). It was also evident that current research on the psychology of body image is basically confined to the quantitative studies of negative body image employing only women participants belonging mostly to the American and Western societies. The present research attempted to develop insights into the basic nature and dynamics of positive body image of the participants by using exploratory research design, a variant of the mixed methods design, which comprises qualitative study followed by a quantitative study.

\section{Study 1: Qualitative Phase}

The first phase of the study aimed to explore the basic themes and descriptive attributes of positive body image with the dual objectives of developing an original understanding the construal of positive body image and prepare a list of relevant items for empirical validation of a scale in the second phase of the study. The major goal of the qualitative study was to find out the basic themes and basic attributes of positive body image to develop a list of preliminary items for the scale.

\section{Methods and Procedure}

The first phase of the study employed a qualitative research design in which semi-structured interviews were conducted using a pre-developed protocol. The contents of the interviews were audio-recorded followed by preparation of verbatim inscriptions suitable for thematic analysis as per the guidelines suggested by Braun and Clarke (2006). Before the actual data collection, a pilot study was conducted to ascertain the procedural details of the qualitative study.

\section{Pilot Study}

A pilot study was conducted recruiting 12 participants out of 51 comprising five male participants age ranging from 22 to 28 year $(\mathrm{M}=25.00, \mathrm{SD}=2.24)$ and seven females age spanning from 23 to $28(\mathrm{M}=$ $25.57, \mathrm{SD}=1.72$ ) pursuing their postgraduate degrees. The data of the pilot study were not included in the final data of the study. The inclusion criteria to choose the participants in the study were: participants with normal Body Mass Index (BMI) 18.50-25 (World Health Organization, 2016), participants with apparent 
normal physical and mental health, participants with age ranging from 20 to 35 years and participants with high scores on Body Appreciation Scale (Avalos et al., 2005).

The interview protocol was prepared after an in-depth study of important research articles on positive body image (Bailey et al., 2015; Frisén \& Holmqvist, 2010; Halliwell, 2015; Swami et al., 2008; Tylka \& Wood-Barcalow, 2015; Tylka, 2013; Webb et al., 2015; Wood-Barcalow et al., 2010). The researchers read these articles thoroughly and prepared a list of major attributes of positive body image. Then, this list of attributes was distributed among three researchers with the request to categorize the contents or the attributes in major categories followed by a conference to develop a consensus about the major attributes of positive body image.

According to the protocol, the interviews of the participants were conducted and the same was also audiotaped with the help of recording device available in the mobile phone version Moto G3. The verbatim inscriptions of the recorded interviews were prepared with comments and the data of all the participants were analysed employing the Thematic Analysis Method (Braun \& Clarke, 2006). The pilot study helped a lot in developing a uniform method of data analysis, assignment of codes and coming up with themes and sub-themes as well as the method of conducting interviews.

\section{The Actual Study}

The following are the major components of the actual qualitative phase of the study:

\section{Sample}

In the first phase of the study, the basic objective was to collect data as per the interview protocol evolved after the pilot study. In this phase, 152 (80 males and 72 females) participants were consulted from which thirty-five comprising 17 males $(\mathrm{M}=26.29, \mathrm{SD}=2.76)$ and 18 females $(\mathrm{M}=24.83, \mathrm{SD}=2.43)$ age ranging from 21-30 year met the inclusion criteria discussed in the pilot study and allowed to take part in the qualitative study (Table 1). The mean body appreciation scores and SDs of the male $(\mathrm{M}=56.82, \mathrm{SD}=$ $4.10)$ and female $(\mathrm{M}=56.33, \mathrm{SD}=4.06)$ participants fell in the inclusion criterion range. In addition, the mean body mass index and SDs of the male $(\mathrm{M}=23.57, \mathrm{SD}=1.04)$ and female $(\mathrm{M}=21.56, \mathrm{SD}=1.51)$ participants also signified normal range. The inclusion and exclusion criteria observed in the pilot study were also followed in the actual qualitative data collection. The details of the age, body appreciation scores, height, weight, and body mass index have been portrayed in Table 1.

\section{Inclusion and Exclusion Criteria}

The inclusion and exclusion criteria observed in the pilot study were also followed in the actual qualitative data collection. Following inclusion criteria were applied to choose the participants and those who did not meet these criteria were excluded from the study:

a. Participants with normal Body Mass Index (BMI) 18.50-25 (World Health Organization, 2016),

b. Participants with apparent normal physical and mental health,

c. Participants with age ranging from 20 to 35 years, and

d. Participants with high scores on the Body Appreciation Scale (Avalos et al., 2005).

\section{Table 1}

Raw scores of age, body appreciation scale, height and weight of the male and female participants of the qualitative study

\begin{tabular}{lll}
\hline S. No. & Male & Female \\
Age & BAS & H
\end{tabular}




\begin{tabular}{lll}
1. & 30 & 52 \\
2. & 28 & 56 \\
3. & 24 & 58 \\
4. & 29 & 61 \\
5. & 27 & 53 \\
6. & 21 & 62 \\
7. & 23 & 55 \\
8. & 29 & 57 \\
9. & 25 & 63 \\
10. & 28 & 61 \\
11. & 26 & 62 \\
12. & 30 & 54 \\
13. & 22 & 52 \\
14. & 28 & 58 \\
15. & 26 & 50 \\
16. & 27 & 53 \\
17. & 24 & 59 \\
18. & - & - \\
\hline
\end{tabular}

Note: BAS- Body Appreciation Scale, H- Height, W - Weight, BMI - Body Mass Index

*Body Mass Index was computed as per the method of World Health Organization (2016).

\section{Tools}

Semi-structured Interview

A protocol was prepared to conduct a semi-structured interview. A set of pertinent questions for conducting the interview was developed by the researcher following the criteria of relevance and significance of the items to the field of positive body image. They were based on existing theories and the conclusions of the important selected studies of the field. The interview protocol prepared in the pilot study was used in this phase for collecting data. The following items were included in the interview protocol of semi-structured interview of the qualitative study:

1. Please describe in detail the way you think and feel about your body?

2. Describe in detail about the importance of positive body image.

3. What significant information you have received from your close relatives (Family, Spouse, Boyfriend/Girlfriend etc.) about your body?

4. In what manner you can achieve a positive image of your body?

5. How does your positive body image influence your social relationships?

6. In your opinion, what type of messages about positive body image should be encouraged by society (TV, Cinema, Magazines, News Papers etc.)?

Body Appreciation Scale

To assess the degree to which participants had positive attitudes towards their bodies, the Body Appreciation Scale (Avalos et al., 2005) was used. The scale was originally in English which was translated first in Hindi followed by retranslation in English by three researchers. The scale consists of 13 items tapping participants' feeling about their body image (e.g., "I respect my body"). Items were rated on a 5-point scale $(1=$ Never, $5=$ Always), and were averaged to arrive at a total score (higher scores reflected greater body 
appreciation). This scale has been widely used by the researchers around the globe for the study of positive body image. The Cronbach's alpha coefficients of the scale have been reported .92 and .94 for male and female, respectively (Tylka, 2013).

Audio Tape Recorder

The verbatim contents of the interviews of the participants were audio recorded with the help of recording device available in the mobile phone version Moto G3. The verbatim inscriptions of the recorded interviews were prepared with comments, if any, to smooth the process of carrying out analysis of the collected data by the judges/experts using the thematic analysis. The data of all the participants were organized according to the order of the items of the interview followed by the analysis employing the thematic analysis method (Braun \& Clarke, 2006).

Body Mass Index (BMI)

The body mass index (BMI) or Quetelet index is a value derived from the mass (weight) and height of an individual. The BMI is defined as the body mass divided by the square of the body height and is universally expressed in units of $\mathrm{kg} / \mathrm{m}^{2}$, resulting from mass in kilograms and height in meters. According to the World Health Organization (2016), the normal range of BMI extends from 18.50 to 25.00.

\section{Procedure}

The first phase of the study employed semi-structured interview according to pre-developed protocol. After the insights developed from conducting the pilot study, some minor modifications were done in the procedure. Following the inclusion of suggestions of three experts, the number of items of the pilot study was reduced from nine to six. All the six items were translated from Hindi to English following translation and retranslation method. The other details of the procedure are the same as were observed in the pilot study.

After having finalized the interview protocol, the participants were recruited. To choose the genuine participants, the inclusion and inclusion criteria were strictly followed. Normal BMI (18.50-25.00), high scores on Body Appreciation Scale (above fifty); apparent physical and mental health and age range from 20 to 35 were the criteria of recruiting the participants in the study. Those participants were allowed to take part in the pilot study who strictly satisfied these criteria. With these preparations, the proposal of the pilot study was put before the Ethical Committee of the Department of Psychology, School of Humanities \& Social Sciences, [BLINDED FOR REVIW] for its approval to conduct the study.

After its approval, the actual data collection was started. Twelve participants were recruited for the study. Before the start of data collection, a rapport was established and the participants were debriefed about the basic objectives of the study. These questions were asked in the style of normal conversation. In addition to these questions, the researchers asked some probe questions in an attempt to clarify the queries arose out of the interview. According to the protocol, the interviews of the participants were conducted and the same was also audio-taped with the help of recording device available in the mobile phone version Moto G3. The verbatim inscriptions of the recorded interviews were prepared with comments, if any, to smooth the process of carrying out the thematic analysis of the collected data by the judges/experts using the thematic analysis. The data of all the participants were organized according to the order of the items of the interview followed by the analysis employing thematic analysis method as per the guidelines of Braun and Clarke (2006) which involves familiarizing with data, generating initial codes, searching for themes, reviewing themes, defining and naming themes and producing the report.

To enhance the reliability and validity of the analysis of qualitative data, to ascertain the procedural details, the efficacy of the items of the interview protocol and to finalize the actual process of data analysis, researchers involved his research supervisor and one more faculty member of the department in the process of data analysis. These three members read and re-read the data and came up with their independent findings regarding the major theme and sub-themes of positive body image. After they had completed data analysis 
and reached to their themes and sub-themes, a conference of the three was held to check and establish consistency of the descriptions, themes and sub-themes inherent in the data. It followed a thorough discussion to reach a consensus about the procedural details and ascertain the efficacy of the procedure and items as well. After the discussion, the researchers agreed on minor modifications in the items of the protocol of the semi-structured interview.

The outcomes of the pilot study proved very important and useful for conducting the actual study. Important modifications, guidelines to be observed in the process of interview, the role of the interviewer, the place and style of interview and many other procedural details were clarified which certainly had positive impacts on the quality of the data that were collected in the actual qualitative study. The number of questions to be queried was reduced from nine to six for the actual study. The method of analysis also got improved especially in the conference. The method of allotting codes was ascertained. The pilot study helped a lot in developing a uniform method of data analysis, allotting codes and coming up with themes and sub-themes as well as the method of conducting interviews.

\section{Results}

Many important attributes of positive body image were generated out after the analysis of the qualitative data. Theme 1 denoted that positive body image is a complex phenomenon which comprises of cognitive, emotional and behavioural components. The scrutiny of the data showed that positive body image has many facets. For example, the participants showed positive thoughts, memory, and decision making regarding their body attributes. Positive body image facilitates people in their satisfaction with life, respecting others and himself/herself helps build confidence and feel inner comfort. Positive body image comes from spirituality; it is not a matter of social comparison. People with joyous nature have a positive outlook towards their body attributes. Positive body image helps shape the mental and physical health. Positive Body image is not only limited to physical fitness but also to the concerns of demeanour.

Theme 2 showed that positive body image is important for self-confidence, success in life and social status. Positive body image of the participants has been found to contribute to enhancing their self-confidence, success and social status. These contributions of positive body image were expressed in many ways. For instance, thinking positively about the body increases self-confidence. The individuals with positive body image do not care much about what others think. Positive body image helps to achieve social status. The positive body image is a source of happiness. Positive comments from close relatives help enhance positive body image. Self-respect towards one's body helps maintain positive body image. Family support enhances the positive experience of the body. Regular health care positively affects body image.

Theme 3 demonstrated that yogic practices, meditation, media and literature help attain positive body image. Positive body image can be cultivated by practising yoga and meditation regularly. Motivational stories, videos etc. help enhance and maintain a positive body image. Positive health practices facilitate achieve a positive body image. Positive media and literature are important for the development of positive body image. Facing negative messages about body image is important for achieving positive body image.

Theme 4 showed that positive body image helps in enhancing and maintaining a relationship. Social relationship plays an important role in maintaining the positive body image. Positive body image helps to get prestige in society. Positive body image helps enhance and maintain relationships in society. Feedback of other person related to the body can affect your relationship. Positive body image helps to attract the opposite gender.

\section{Discussion}

The results of the qualitative study have evinced that positive body image is a complex phenomenon which is shaped and regulated by an intricate interplay of personality attributes, interactions, emotions, cognitions, multiple outcomes and social influences. The findings of the study demonstrated that there were 
four major themes with many sub-themes which emerged out the thematic analysis of the qualitative data. The theme 1 signifies that positive body image led to positive thoughts, memory and decision making of the participants of both the gender. Theme 2 denoted that the participants with perceptions of positive body image reported self-confidence, success in life and social status. Theme 3 indicated that positive health practices such as yogic practices and meditation had important contributions in managing their positive body image. Theme 4 denoted that positive body image helped the participants of both the sex to develop, maintain and enhance interpersonal and social relationships.

The results obtained in the present study find sufficient support from previous studies conducted on positive body image. Previous studies have reported that body image is an inseparable constituent of self-concept affecting human functioning, performance and quality of life (Cash \& Smolak, 2011). Positive body image has also been reported to regulate and impact interpersonal relationships, self-esteem, eating behaviours, well-being and other dimensions of human behaviours irrespective of age, gender, cultural background and personality attributes of the individuals in previous studies (Cash \& Smolak, 2011). Like previous findings, the present study reported that positive body image signified perceptual, affective, cognitive and behavioural body images constituting four major dimensions of positive body image (National Eating Disorder Association, 2018). The results of the present also exhibited that individuals possessing positive body image exhibit a clear, true perception of body shape, appreciate their natural body shape, feel proud and accept the uniqueness of their body (National Eating Disorder Association, 2018).

Many positive attributes and outcomes have been reported by the previous researchers carrying positive body image. Similar to the previous findings, the present study had shown that positive body image entailed optimism, self-esteem, social support, adaptive coping and weight stability. The major themes and subthemes of the present study have many similarities with the themes reported by previous researchers. A close examination of the qualitative data of the present study has evinced that many positive outcomes were reported to be carried with having a positive body image which gets supported from previous studies (Lyubomirsky \& Lepper, 1999; Swami et al., 2008, 2013). Similar to the previous findings, the present study also corroborated that the desire to lose weight, look beautiful, healthy and energetic with productive and good performance was closely associated with the positive body image which, in turn, shape functioning and performance of the individuals (Chaiton et al., 2009). Previous studies have also reported a variety of components of positive body image which included body appreciation, body acceptance, inner positivity influencing outer demeanour, a broad conceptualization of beauty, media literate, unconditional acceptance from others, spirituality/religion and listening to and taking care of the body (Wood-Barcalow et al., 2010b). Thus, four themes along with many sub-themes emerged from the analysis of the qualitative data fulfilled the first and big goal of the present study.

\section{Study 2: Quantitative Phase}

The results of the qualitative phase of the study indicated that the construct of positive body image is multi-faceted and multi-dimensional in nature, different from the negative body image, characterized by different attributes and regulatory mechanisms, closely associated with a host of measures namely body appreciation, positive rational acceptance coping, body image flexibility, body functionality, attunement, body pride, positive and self-accepting body talk, body sanctification, a broad conceptualization of beauty and body acceptance by others (Webb et al., 2015). The positive body image has been reported to play a significant role in positive human functioning and productivity proliferating across age, gender and cultures (Webb et al., 2015). In this backdrop, the quantitative phase of the study was planned in consonance with the insights emerged from the qualitative study. This phase of study attempted to prepare a list of relevant items for further establishment of a scale of positive body image for adults of the Indian population. 


\section{Objectives}

1. To prepare items for positive body image scale from the descriptions of the qualitative data, and

2. To standardize a comprehensive tool to measure positive body image based on the themes and descriptions of the qualitative data,

\section{Methods and Procedure}

The major goal of the quantitative phase of the study was to prepare items for positive body image scale and establish empirically its reliability and validity. Initially, a list of 110 items was prepared followed by face validation of the items by three experts who have sufficient background in psychological theorizing, measurement and research. After face validation, only 45 items were retained for empirical validation on an adult sample.

\section{Sample}

In the quantitative phase of the study, 507 participants comprising 268 males $(\mathrm{M}=26.29, \mathrm{SD}=2.75)$ and 239 females $(\mathrm{M}=24.83, \mathrm{SD}=2.43)$ age ranging from 20-35 years pursuing their undergraduate and postgraduate studies from different educational institutions of Sagar, Madhya Pradesh, India were recruited. The convenient sampling procedure was used to select the participants for the study.

\section{Tools}

General Health Questionnaire

To measure the general health of the participant, General Health Questionnaire (Goldberg \& Hillier, 1979) was used. The participants were asked to compare their recent psychological state with their usual state. For each item, four answer possibilities are available. The higher the score the poorer was the psychological well-being.

Quality of Life

Quality of life of the participants was assessed through the WHO Quality of Life inventory (World Health Organization, 1996). This questionnaire comprises 26 items. It entails four domains: physical quality of life, psychological quality of life, social quality of life and the environmental quality of life. The scoring pattern was based on a five-point scale.

\section{Procedure}

After the procurement of the psychometric tools, the participants were approached individually and debriefed about the basic goals of the study. Then the participants were supplied with a set of the scales. Participants were instructed to read the instructions carefully before giving the responses of any questionnaire. The mean, standard deviation (SD), t-test, Pearson Product Moment Method of Correlation and factor analysis were carried out on the data.

\section{Results}

The central aims of the second phase of the study were to estimate the reliability and validity of the positive body image scale by assessing its association with the scores of general health and quality of life. Results have presented in three sections. As per the method discussed above, the descriptions of the contents of the inscriptions were prepared. On the basis of these descriptions, a list of 50 items was prepared initially. Three experts were engaged to ascertain the face validity of the items. A pool of items was generated on the basis of emerged themes and attributes of positive body image. Initially, 50 items were written in Hindi language and to assess the face validity of the scale again three experts have critically 
scrutinized all the 50 items and finally, 30 items were found suitable to go for empirical validation. All the items were rated on a seven-point scale (1-Very Strongly Disagree, 2-Strongly Disagree, 3-Somewhat Disagree, 4-Neutral, 5-Somewhat Agree, 6-Strongly Agree, 7-Very Strongly Agree).

To ascertain dimensional attributes of the positive body image scale, the scale was administered on five hundred seven adults. The factor structure was computed by the principal component analysis with Varimax Rotation Method was computed. Six items were excluded from the scale due to their poor loadings. The details of the values of factor loadings of the 30 items have been presented in Table 2 .

Table 2

\section{Factor loadings of the items of the Positive Body Image Scale}

\begin{tabular}{lll}
\hline S. No. & Items & Factor Loadings \\
I & II & .769 \\
1. & I respect my body and its parts. & .763 \\
2. & I feel good about my body. & .557 \\
3. & I do not compare my body and its parts to anyone else. & .547 \\
4. & My body is attractive. & .691 \\
5. & I am satisfied with my body. & .757 \\
6. & I think exercise is essential to keep the body healthy. & .754 \\
7. & I want people to accept my body as it is. & .609 \\
8. & My body makes me self-confident. & .633 \\
9. & My body makes me happy. & .499 \\
10. & I have so many good qualities in my body. & .355 \\
11. & My body has an important contribution to become successful in my life. \\
12. & I think physical beauty has great importance in life. & -.006 \\
13. & Positive body image is helpful in the adjustment. & .347 \\
14. & My body is a gift given by God. & .401 \\
15. & The image of my body is helpful in making social relations. & .157 \\
16. & My body image enhances my personality. & .162 \\
17. & My body attracts people. & .194 \\
18. & The image of my body is helping me in attaining my social status. \\
19. & My body image helps me to take advantage of opportunities in different areas. & .068 \\
20. & People respond positively to my body. & .112 \\
21. & I am conscious and aware of my body. & .131 \\
22. & I follow the systematic routine to maintain my body image. \\
23. & I think Sport/Gaming is important to maintain the body image. \\
24. & I enjoyed being part of social gatherings. & .225 \\
\hline & & .278 \\
\hline
\end{tabular}

After the factor analysis of the items of the scale, two major factors of positive body image emerged. After close consideration of the contents of items of each factor, they were labelled as body appreciation and body effectiveness. The details of the two components are as under:

\section{Body Appreciation}

The items of Factor 1 were labelled as body appreciation. The body appreciation referred to the respect, satisfaction, acceptance, positive feelings, attractiveness, self-enhancing effects and God-giftedness of body and its attributes. This name was appropriate as it involved respect for the body, good feeling, noncomparison, attractiveness, high satisfaction with the body and its attributes, promotive aspects (exercise), desire for acceptance of real body and its attributes by others, enhancing (self-confidence, happiness), positive perception (many good qualities) and a gift of God. These attributes of positive body image were also evident in the verbalizations of the participants of the qualitative study. It consisted of 11 items. 


\section{Body Effectiveness}

This body effectiveness dimension consists of the items that consisted of the effectiveness of body in achieving various life success and outcomes and helpful in achieving social praise, opportunities, positive feedback, identity as well as positive efforts. This label was appropriate as body effectiveness was reported to be helpful in life success, adjustment, beauty, effective social relationships, effective personality, attractive capacity, social praise and status, life opportunities, positive responses of others, familiarity, positive efforts (systematic routine, Sport/Gaming) and positive social and interpersonal outcomes of the participants.

\section{Psychometric Properties}

The reliability and validity of the components and overall scale were estimated. The details are as under:

\section{Reliability}

The scale comprises of two dimensions of positive body image: body appreciation and body effectiveness comprising 11 items and 13 items, respectively. The reliability was estimated by computing Cronbach's Alpha which was $.878, .844$ and .903 body appreciation, body effectiveness and overall positive body image scale, respectively

Validity

Its validity was estimated by computing correlations among the scores of the body appreciation, body effectiveness and overall positive body image, and the scores of physical quality of life, psychological quality of life, social relation quality of life, environmental quality of life and overall quality of life. In addition, the scores of body appreciation body effectiveness and overall positive body scale were correlated with the scores of and somatic, depression and overall general health.

The scores of body appreciation exhibited significant positive correlations with the scores of physical $(\mathrm{r}=$ $.231, p=.000)$, psychological $(\mathrm{r}=.299, p=.000)$, social relation $(\mathrm{r}=.157, p=.010)$ and environmental $(\mathrm{r}=.226, p=.000)$ dimensions of quality of life of the male participants. Likewise, the scores of the female participants on body appreciation and the scores of physical $(\mathrm{r}=.290, p=.000)$, psychological $(\mathrm{r}=.411, p$ $=.000)$, social relation $(\mathrm{r}=.175, p=.007)$ and environmental $(\mathrm{r}=.308, p=.000)$ aspects of quality of life also exhibited significant positive correlations. Similarly, the overall scores on this measure demonstrated significant positive correlations with the scores of physical $(\mathrm{r}=.235, p=.000)$, psychological $(\mathrm{r}=.336, p=$ $.000)$, social relation $(\mathrm{r}=.174, p=.000)$ and environmental $(\mathrm{r}=.240, p=.000)$ aspects of quality of life.

The results showed that there were significant positive correlations among the scores of body effectiveness and the scores physical $(\mathrm{r}=.298, p=.000)$, psychological $(\mathrm{r}=.291, p=.000)$, social relation $(\mathrm{r}=.218, p$ $=.000)$ and environmental $(\mathrm{r}=.196, p=.001)$ aspects of quality of life of the males. The same trend also got repeated in the case of females again where this dimension of positive body image showed significant positive correlations with scores of the physical $(\mathrm{r}=.374, p=.000)$, psychological $(\mathrm{r}=.303, p=.000)$, social relation $(\mathrm{r}=.188, p=.004)$ and environmental $(\mathrm{r}=.226, p=.000)$ aspects of quality of life. Similarly, significant positive correlations were also observed among the scores of body effectiveness and the scores of physical $(\mathrm{r}=.329, p=.000)$, psychological $(\mathrm{r}=.316, p=.000)$, social relation $(\mathrm{r}=.212, p=.000)$ and environmental $(\mathrm{r}=.218, p=.000)$ aspects of quality of life.

The results showed that there were significant positive correlations among the scores of overall positive body image and the scores of physical $(\mathrm{r}=.300, p=.000)$, psychological $(\mathrm{r}=.329, p=.000)$, social relation $(\mathrm{r}=.213, p=.000)$ and environmental $(\mathrm{r}=.234, p=.000)$ aspects of quality of life of the males. The significant positive correlations among the scores of overall positive body image were also observed with the scores of physical $(\mathrm{r}=.374, p=.000)$, psychological $(\mathrm{r}=.303, p=.000)$, social relation $(\mathrm{r}=.188, p$ $=.004)$ and environmental $(\mathrm{r}=.226, p=.000)$ aspects of quality of life of the females. Lastly, significant positive correlations among the scores of overall positive body image and the scores of physical $(\mathrm{r}=.329$, 
$p=.000)$, psychological $(\mathrm{r}=.316, p=.000)$, social relation $(\mathrm{r}=.212, p=.000)$ and environmental $(\mathrm{r}=$ $.218, p=.000$ ) dimensions of quality of life were observed for all the participants.

Contrarily, significant negative correlations were found among the scores of body appreciation and the scores of somatic $(\mathrm{r}=-.138, p=.024)$, depression $(\mathrm{r}=-.121, p=.048)$, general health $(\mathrm{r}=-.167, p=.006)$ of the male participants. For the females, the results showed similar trend as there were also significant negative correlations among the scores of body appreciation and the scores of depression $(\mathrm{r}=-.127, p=.05)$ and general health $(\mathrm{r}=-.191, p=.003)$ except somatic $(\mathrm{r}=-.119, p=.066)$. These values have been presented in Table 3.

\section{Table 3}

Coefficients of correlation among the scores of various components of positive body image, quality of life and general health of the male and female participants

\begin{tabular}{ll}
\hline S. No. & Measures \\
Male & Female \\
r & $p$ \\
1. & Body Appreciation and Physical Quality of Life \\
Body Appreciation and Psychological Quality of Life & $.299^{* *}$ \\
Body Appreciation and Social Relation Quality of Life & $.157^{* *}$ \\
Body Appreciation and Environmental Quality of Life & $.226^{* *}$ \\
Body Appreciation and Somatic Symptoms & $-.138^{*}$ \\
Body Appreciation and Depression & $-.121^{*}$ \\
Body Appreciation and Overall General Health & $-.167^{* *}$ \\
2. & Body Effectiveness and Physical Quality of Life \\
Body Effectiveness and Psychological Quality of Life & $.291^{* *}$ \\
Body Effectiveness and Social Relation Quality of Life & $.218^{* *}$ \\
Body Effectiveness and Environmental Quality of Life & $.196^{* *}$ \\
Body Effectiveness and Somatic Symptoms & -.018 \\
Body Effectiveness and Depression & -.028 \\
Body Effectiveness and Overall General Health & -.055 \\
3. & Overall Positive Body Image and Physical Quality of Lif \\
Overall Positive Body Image and Psychological Quality of Life & $.329^{* *}$ \\
Overall Positive Body Image and Social Relation Quality of Life & $.213^{* *}$ \\
Overall Positive Body Image and Environmental Quality of Life & $.234^{* *}$ \\
Overall Positive Body Image and Somatic Symptoms & -.081 \\
Overall Positive Body Image and Depression & -.079 \\
Overall Positive Body Image and Overall General Health & -.118 \\
\hline
\end{tabular}

$\left({ }^{*} p<.05,{ }^{* *} p<.01\right)$

The overall scores body image of the participants showed the same trend as these scores exhibited again significant negative correlations with the scores of somatic $(\mathrm{r}=-.140, p=.002)$, depression $(\mathrm{r}=.134$, $p=.004)$ and $(\mathrm{r}=.178, p=.000)$ general health. There were negative correlations among the scores of body effectiveness and the scores of somatic $(\mathrm{r}=-.018, p=.772)$, depression $(\mathrm{r}=-.028, p=.643)$ and general health $(\mathrm{r}=-.055, p=.369)$ of the males. For the female participants, non-significant negative correlations were also observed among the scores of body appreciation and the scores of somatic $(\mathrm{r}=-.036, p=.58)$, depression $(\mathrm{r}=-.043, p=.504)$ and $(\mathrm{r}=-.096, p=.138)$ general health. The same trend were observed for all the participants where non-significant negative correlations were again recorded for the scores of this measure and the scores of somatic $(\mathrm{r}=.045, p=.325)$, depression $(\mathrm{r}=.003, p=.947)$ and general health $(\mathrm{r}=.031, p=.508)$. 
The negative significant correlations were observed among the scores of positive body image and the scores of general health of the male $(\mathrm{r}=-.118, p=.054)$, female $(\mathrm{r}=-.156, p=.016)$ and all the participants $(\mathrm{r}=-.108, p=.019)$. The significant negative correlation was also recorded between the scores of positive body image and somatic $(\mathrm{r}=-.098, p=.033)$ dimension of the general health of all the participants. The scores of positive body image and the scores of somatic $(\mathrm{r}=-.081, p=.189)$ and depression $(\mathrm{r}=-.079, p$ $=.200)$ of the males exhibited non-significant negative correlations. The same were also observed for scores of positive body image of the females and their scores on somatic $(\mathrm{r}=-.082, p=.205)$ and depression $(\mathrm{r}=$ $-.091, p=.161)$ aspects of general health. Additionally, a non-significant negative correlation was observed between the scores of positive body image and depression $(\mathrm{r}=-.065, p=.158)$ of all the participants.

\section{Discussion}

The findings of Study 2 demonstrated that the two factors emerged after the factor analysis of the data of positive body image namely, body appreciation and body effectiveness with 11 and 13 items, respectively. Body appreciation reflected the respect, satisfaction, acceptance, positive feelings, attractiveness, self-enhancing effects and God-giftedness of body and its attributes. Similarly, body effectiveness denoted the effectiveness of body in achieving various life success and outcomes and helpful in achieving social praise, opportunities, positive feedback, identity as well as positive efforts. The coefficients of reliability of the two components and the entire scale were computed by Cronbach's Alpha that indicated satisfactory levels of statistical significance. The coefficients of the validity of the scale were computed by computing the coefficients of correlations among the scores of two components and the overall positive body image and the scores of quality of life (World Health Organization, 1996) and the scores of general health questionnaire (Goldberg \& Hillier, 1979; Parihar et al., 2019).

The findings of study 2 showed that the scores of body appreciation, body effectiveness and overall positive body image exhibited significant positive correlations with the scores of physical, psychological, social relation and environmental dimensions of quality of life of the male and female participants. Irrespective of gender, the scores body appreciation, body effectiveness and overall positive body image demonstrated significant positive correlations with the scores of physical, psychological, social relation and environmental aspects of quality of life. Contrarily, significant negative correlations were found among the scores of body appreciation and the scores of somatic, depression, general health of the male and female participants except for somatic symptoms for females. These findings were also true when all the data of male and female participants on various measures were pooled.

There were significant negative correlations among the scores of body appreciation and the scores of somatic symptoms, depression and general health of the male and female and all the participants except between the scores of body appreciation and somatic symptoms of the female participants for which a non-significant correlation was observed. The non-significant negative correlations were recorded among the scores of body effectiveness and the scores of somatic symptoms, depression and general health of the male and female and all the participants. Contrarily, the scores of overall positive body image, and the scores of somatic symptoms and depression of the male, female and all the participants except between the scores of overall positive body image and somatic symptoms of all the participants. The significant negative correlations were observed among the scores of overall positive body image and the scores of the general health of the male, female and all the participants. The findings evinced that positive body image has a significant role in shaping the quality of life and general health of the male and female participants.

\section{General Discussion}

Measurement of positive body image has been recognized as an important task before researchers (Webb et al., 2015). Several positive body image scales have been developed and the empirical evidence suggested that none of these tools was derived the attributes of positive body image using in-depth qualitative study. 
Therefore, it necessitated developing a comprehensive measure of positive body image. To this end, it was aimed to develop a scale on positive body image adopting the exploratory design. The findings of the first phase of the study facilitated to emerge four significant themes with a set of relevant attributes of positive body image. The findings of the qualitative study evinced that positive body image significantly shapes the nature of thoughts, memory and decision making of the participants of both the gender. Moreover, these perceptions of positive body image, in turn, structure many positive life outcomes such as self-confidence, success in life and social status. It was also evident that positive health practices such as yogic practices and meditation extend significant contributions in managing the nature and dynamics of positive body image. In addition, positive body image facilitate to participants of both the sexes to develop, maintain and enhance interpersonal and social relationships. Many descriptive attributes of positive body image have emerged in the first phase of the study that acted as the major background for preparing the items of the scale.

The findings of the first phase of the study also evinced the positive body image to be associated with many positive life outcomes. The findings of the present study have been mirrored in many previous studies that have suggested similar benefits of having a positive body image. For example, the researchers have suggested a positive body image to be significant to facilitate inner resources for optimizing health and well-being for all sorts of people (Cook-Cottone, 2013). The close association between body image and psychological wellbeing such as self-esteem, life-satisfaction and stress have also been observed (Peternel \& Sujoldžić, 2009). Swami et al. (2013) have suggested that body image and subjective happiness are closely associated as body appreciation is a strong predictor of subjective happiness. Contrarily, a lower positive body image score may have negative consequences for the quality of life (Cash, 2008; Feingold \& Mazzella, 1998; Garner, 1997).

The major drives behind body image affecting human functioning are people's desire to lose weight; look beautiful, healthy and energetic with productive and good performance. The positive body image and dissatisfaction have been reported to play an important role in the development of obesity, depression and psychological disturbances of various sorts. The perceptions of body image have been reported to be associated with obesity and depression (Chaiton et al., 2009). Moreover, positive body image has also been reported to be associated with other significant dimensions of human functioning in some Indian studies. For example, chronic health conditions (Pankaj Jain \& Tiwari, 2016a; Tiwari \& Kumar, 2015), women identity (Priya Jain, Tiwari, \& Awasthi, 2018) and self-construal of adolescent girls (Priya Jain, Tiwari, Awasthi, et al., 2018) have been observed to be linked with positive body image.

Moreover, the nature of body image perceptions has been reported to be linked with a number of healthy and unhealthy behaviours. It has been argued that higher positive body image may cause people to internalize positive health behaviours (Grogan, 2008) and engage in sports activities (Burgess et al., 2006; Choi, 2005). The differences in the mean scores of positive body image, quality of life and general health of the participants with neuroticism and extraversion can be explained in terms of differences in their basic cognitive, emotional and behavioural predispositions reported by previous researchers. It has been argued that dissimilarities in personality traits give birth to a difference in the patterns of affect, behaviour, cognition and desires (Ortony et al., 2005) and environmental responsivity (Corr, 2008; Revelle, 1995). These mentions evince that positive body image carries significant implications to understand a host of positive and negative outcomes of life.

The second phase of the study was conducted with the goal to develop and standardize a scale of positive body image for the Indian adults. The quantitative phase of the study led to emerging (Study 2) a scale comprising 24 items with two components: body appreciation (11 items) and body effectiveness (13 items). Body appreciation reflected the respect, satisfaction, acceptance, positive feelings, attractiveness, self-enhancing effects and God-giftedness of body and its attributes. Likewise, body effectiveness demonstrated the effectiveness of the body in achieving various life successes such as social praise, opportunities, positive feedback, identity and positive efforts. The findings also showed reliability coefficients to be satisfactory for the two components and overall scale. In addition, its validity was also satisfactory that was estimated by computing coefficients of correlation between the scores of the two components and the overall positive body image and the scores of general health questionnaire (Goldberg \& Hillier, 1979) and quality of 
life (World Health Organization, 1996). It predictive strength was verified in a recent study employing the same measure of general health on Indian participants (Parihar et al., 2019).

\section{Implications and Limitations}

The findings of the study may have important theoretical and practical implications for the field of body image, school interventions, Psychology of adolescents and sportspersons and mental health interventions. In addition, the findings may also find their implications for promotive and preventive health purposes. The study has also been marred by some limitation like other scientific endeavours. For example, lack of diverse groups of participants, small sample for the qualitative study, participants from a single culture, only adult age group and a limited number of variables for quantitative phase of the study were some of the limitations of the study.

\section{References}

Avalos, L., Tylka, T. L., \& Wood-Barcalow, N. (2005). The Body Appreciation Scale: Development and psychometric evaluation. Body Image, 2(3), 285-297. https://doi.org/10.1016/j.bodyim.2005.06.002

Bailey, K. A., Gammage, K. L., van Ingen, C., \& Ditor, D. S. (2015). "It's all about acceptance": A qualitative study exploring a model of positive body image for people with spinal cord injury. Body Image, 15, 24-34. https://doi.org/10.1016/j.bodyim.2015.04.010

Braun, V., \& Clarke, V. (2006). Using thematic analysis in psychology. Qualitative Research in Psychology, 3(2), 77-101. https://doi.org/10.1191/1478088706qp063oa

Brown, T. A., Cash, T. F., \& Mikulka, P. J. (1990). Attitudinal Body-Image Assessment: Factor Analysis of the Body-Self Relations Questionnaire. Journal of Personality Assessment, 55(1-2), 135-144. https://doi.org/10.1080/00223891.1990.9674053

Burgess, G., Grogan, S., \& Burwitz, L. (2006). Effects of a 6-week aerobic dance intervention on body image and physical self-perceptions in adolescent girls. Body Image, 3(1), 57-66. https://doi.org/10.1016/j.bodyim.2005.10.005

Cash, T. F. (2002). The situational inventory of body-image dysphoria: Psychometric evidence and development of a short form. International Journal of Eating Disorders, 32(3), 362-366. https://doi.org/10.1002/eat.10100

Cash, T. F. (2008). The body image workbook: An eight-step program for learning to like your looks (2nd ed). New Harbinger Publications.

Cash, T. F., \& Smolak, L. (Eds.). (2011). Body image: A handbook of science, practice, and prevention (2nd ed). Guilford Press.

Chaiton, M., Sabiston, C., O'Loughlin, J., McGrath, J. J., Maximova, K., \& Lambert, M. (2009). A structural equation model relating adiposity, psychosocial indicators of body image and depressive symptoms among adolescents. International Journal of Obesity, 33(5), 588-596. https://doi.org/10.1038/ijo.2009.43

Choi, P. Y. L. (2005). Femininity and the Physically Active Woman (1st ed.). Routledge. https://doi.org/10.4324/9780203977606

Cook-Cottone, C. P. (2013). Healthy eating in schools: Evidence-based interventions to help kids thrive (1st ed). American Psychological Association.

Corr, P. J. (2008). The reinforcement sensitivity theory. In P. J. Corr (Ed.), The reinforcement sensitivity theory of personality (First, pp. 347-376). Cambridge University Press. 
Creswell, Clark, Gutmann, \& Hanson. (2003). Advanced mixed methods research designs. In Handbook of mixed methods in social and behavioral research (pp. 209-240). Thousand Oaks.

Creswell, J. W. (2004). Designing A Mixed Methods Study In Primary Care. The Annals of Family Medicine, 2(1), 7-12. https://doi.org/10.1370/afm.104

Feingold, A., \& Mazzella, R. (1998). Gender Differences in Body Image Are Increasing. Psychological Science, $9(3)$, 190-195. https://doi.org/10.1111/1467-9280.00036

Franzoi, S. L., \& Shields, S. A. (1984). The Body Esteem Scale: Multidimensional Structure and Sex Differences in a College Population. Journal of Personality Assessment, 48(2), 173-178. https://doi.org/10.1207/s15327752jpa4802_12

Frisén, A., \& Holmqvist, K. (2010). What characterizes early adolescents with a positive body image? A qualitative investigation of Swedish girls and boys. Body Image, 7(3), 205-212. https://doi.org/10.1016/j.bodyim.2010.04.001

Garner, D. M. (1997). Body Image in America: Survey Results. Psychology Today. http://www.psychologytoday.com/articles/199702/body-image-in-america-survey-results

Goldberg, D. P., \& Hillier, V. F. (1979). A scaled version of the General Health Questionnaire. Psychological Medicine, 9(1), 139-145. https://doi.org/10.1017/s0033291700021644

Grogan, S. (2008). Body image: Understanding body dissatisfaction in men, women, and children (2nd ed). Routledge.

Halliwell, E. (2015). Future directions for positive body image research. Body Image, 14, 177-189. https://doi.org/10.1016/j.bodyim.2015.03.003

Holmqvist, K., \& Frisén, A. (2012). "I bet they aren't that perfect in reality:" Appearance ideals viewed from the perspective of adolescents with a positive body image. Body Image, 9(3), 388-395. https://doi.org/10.1016/j.bodyim.2012.03.007

Jain, Pankaj, \& Tiwari, G. K. (2016a). Body image satisfaction and Life Satisfaction in HIV/AIDS patients. The International Journal of Indian Psychology, 3(2, 1), 85-90. https://doi.org/10.25215/0302.010

Jain, Pankaj, \& Tiwari, G. K. (2016b). Positive body image and general health: A Mixed Methods Study. The International Journal of Indian Psychology, 4 (1, 76), 33-51. https://doi.org/10.25215/0476.003

Jain, Priya, Tiwari, G. K., \& Awasthi, I. (2018). Menstrual distress and attitude towards femininity of rural and urban adolescent girls. Madhya Bharti, 74, 222-233.

Jain, Priya, Tiwari, G. K., Awasthi, I., \& Chaubey, A. (2018). Menstrual Experiences of Adolescent Girls: A Qualitative Study. The International Journal of Indian Psychology, 6(1), 74-82. https://doi.org/10.25215/0601.087

Lyubomirsky, S., \& Lepper, H. S. (1999). A Measure of Subjective Happiness: Preliminary Reliability and Construct Validation. Social Indicators Research, 46(2), 137-155. https://doi.org/10.1023/A:1006824100041

Mendelson, B. K., Mendelson, M. J., \& White, D. R. (2001). Body-Esteem Scale for Adolescents and Adults. Journal of Personality Assessment, 76(1), 90-106. https://doi.org/10.1207/S15327752JPA7601_6

National Eating Disorder Association. (2018, February 18). Body Image. National Eating Disorders Association. https://www.nationaleatingdisorders.org/body-image-0

Ortony, A., Norman, D. A., \& Revelle, W. (2005). Effective functioning: A three level model of affect, motivation, cognition, and behavior. In J. Fellous 83 M. Arbib (Eds.), Who needs emotions? The brain meets the machine (pp. 173-202). Oxford University Press. 
Parihar, P., Pandey, R., Tiwari, G. K., Mishra, M., \& Rai, P. K. (2019). Assessing the protective strengths of positive body image for somatic symptoms, anxiety, social dysfunction and depression. The European Journal of Counselling Psychology (under Review).

Peternel, L., \& Sujoldžić, A. (2009). Adolescents Eating Behavior, Body Image and Psychological Well-Being. Collegium Antropologicum, 33(1), 205-212.

Revelle, W. (1995). Personality Processes. Annual Review of Psychology, 46(1), 295-328. https://doi.org/10.1146/annurev.ps.46.020195.001455

Swami, V., Hadji-Michael, M., \& Furnham, A. (2008). Personality and individual difference correlates of positive body image. Body Image, 5(3), 322-325. https://doi.org/10.1016/j.bodyim.2008.03.007

Swami, V., Tran, U. S., Brooks, L. H., Kanaan, L., Luesse, E.-M., Nader, I. W., Pietschnig, J., Stieger, S., \& Voracek, M. (2013). Body image and personality: Associations between the Big Five Personality Factors, actual-ideal weight discrepancy, and body appreciation: Body Image and Personality. Scandinavian Journal of Psychology, 54(2), 146-151. https://doi.org/10.1111/sjop.12014

Tiwari, G. K., \& Kumar, S. (2015). Psychology and body image: A review. Shodh Prerak, 5(1), 1-9.

Tylka, T. L., \& Wood-Barcalow, N. L. (2015). What is and what is not positive body image? Conceptual foundations and construct definition. Body Image, 14, 118-129. https://doi.org/10.1016/j.bodyim.2015.04.001

Tylka, Tracy L. (2013). Evidence for the Body Appreciation Scale's measurement equivalence/invariance between U.S. college women and men. Body Image, 10(3), 415-418. https://doi.org/10.1016/j.bodyim.2013.02.006

Webb, J. B., Wood-Barcalow, N. L., \& Tylka, T. L. (2015). Assessing positive body image: Contemporary approaches and future directions. Body Image, 14, 130-145. https://doi.org/10.1016/j.bodyim.2015.03.010

Wood-Barcalow, N. L., Tylka, T. L., \& Augustus-Horvath, C. L. (2010a). "But I Like My Body": Positive body image characteristics and a holistic model for young-adult women. Body Image, 7(2), 106-116. https://doi.org/10.1016/j.bodyim.2010.01.001

Wood-Barcalow, N. L., Tylka, T. L., \& Augustus-Horvath, C. L. (2010b). "But I Like My Body": Positive body image characteristics and a holistic model for young-adult women. Body Image, 7(2), 106-116. https://doi.org/10.1016/j.bodyim.2010.01.001

World Health Organization. (1996). WHOQOL-BREF: Introduction, administration, scoring and generic version of the assessment: field trial version, December 1996. https://apps.who.int/iris/handle/10665/63529

World Health Organization. (2016). Body mass index-BMI. World Health Organization. http://www.euro.who.int/en/health-topics/disease-prevention/nutrition/a-healthy-lifestyle/body-massindex-bmi 